\title{
Why OSA may be Regarded as the Real Hidden Killer in Diabetes
}

\author{
Tatti $\mathrm{P}^{1 *}$, Strollo $\mathrm{F}^{2}$, Politi $\mathrm{L}^{3}$ and Passali $\mathrm{D}^{3}$ \\ ${ }^{1}$ Diabetes and Endocrinology Unit, ASL RMH, Roma, Italy \\ ${ }^{2}$ Diabetes and Metabolism Unit, Santo Spirito Hospital, Roma, Italy \\ ${ }^{3}$ ENT Unit, University of Siena, Italy
}

Received: January 07, 2014; Accepted: February 03, 2014; Published: February 05, 2014

${ }^{*}$ Corresponding author: Patrizio Tatti, Diabetes and Endocrinology Unit, ASL RMH-Roma, Italy, E-mail: info@patriziotatti.it

\begin{abstract}
Obstructive Sleep Apnea Syndrome (OSAS) is a common and usually overlooked phenomenon causing a deep reduction in oxygen delivery to the body tissues and a serious disruption of the sleep pattern. Furthermore there are scattered reports in the medical literature pointing to a role of this respiratory disturbance on sudden death. We hypothesize that the phenomenon may have a role in the vascular damage and the high cardiovascular death rate of diabetes interacting at least at three consecutive steps.
\end{abstract}

Keywords: OSAS; Diabetes; Sudden death

\section{Introduction}

It is well known from the literature that subjects with diabetes are at an increased risk for premature cardiovascular death [1]. Although this has been definitely demonstrated, the correction of hyperglycemia does not seem to reduce the risk. Rather the benefit of this intervention versus a reduction of cholesterol or of blood pressure ranks very low $[2,3]$ and under certain circumstances may even be definitely harmful [4]. Throughout the years many other supposed risk factors that may concur to this increased burden of deaths in diabetes have been identified, [1] like central obesity, hypercholesterolemia, low HDL cholesterol, other lipid abnormalities, hypertension, smoking, psychological factors, hypercystinemia. More recently the high variability of blood glucose has been under close scrutiny for its potential role in increased free radical formation [5]. However no one of these risk factor was conclusively proven to be the real cause of the increased death risk. In this paper we examine the hypothesis that the respiratory disturbance occurring during sleep, known as Obstructive Sleep Apnea Syndrome (OSAS), an apparently unrelated condition, may act lethally, in subsequent steps, concurring to predispose to atherosclerosis, and acting as the final killer through oxygen deprivation. The prevalence of this condition in diabetics varies from 58 to $86 \%$ [6-9]. Many studies pointed out a role of this respiratory disturbance on diabetes incidence [10] and glucose control [8,11], and a recent statement of the EASD emphasized this topic [12]. The impact of OSAS on blood glucose control is probably obscured by the close association of this phenomenon with sleep disturbance. In fact other studies demonstrated that alterations of the sleep architecture can interfere with glucose metabolism [13-16]. We have recently demonstrated that the number of nocturnal awakenings is related to the instability of the fasting blood glucose [17].

We hypothesize that the presence of OSAS may have long term and immediate consequences on the cardiovascular system (Figure 1) through multiple mechanisms.

\section{Viewpoint}

We surmise that the responsibility of premature Atherosclerosis seen in the diabetic may be, at least in part, attributed to disturbed sleep/oxygen deprivation of OSAS. This chronic inflammatory condition, superimposed on the coexistent cardiovascular damage, aggravates diabetes control and hypertension, setting the stage for the cardiovascular catastrophe, which will be precipitated by the apnoeic/hypoxic episodes.

\section{Evidence from the literature}

We searched Pub med for all the papers including the terms "OSAS diabetes" and obtained 122 titles. When we added the term "death" we obtained only 3 papers. The search "OSAS sudden death" returned 30 papers, and when we added the term "diabetes" we again went down to 3 . Out of these papers we selected those with long term follow up, a population of $>50$ subjects, and, when experimental data were required to support the theory, we selected those with the use of more sophisticated technology. Many papers have linked the alteration of sleep architecture and OSAS to impaired glucose tolerance, diabetes, increased progression to diabetes, insulin resistance, absence of the first peak insulin response to IV glucose, other metabolic disturbances, hypertension, stroke, cardiovascular death [18]. Furthermore OSAS may induce pathophysiological changes that predispose to impaired cardiovascular function [19]. Recently we also demonstrated a role of the number of nocturnal awakenings on the increased variability of the fasting blood glucose levels [17]. In a subsequent study with the use of polysomnographic 
Step \#1

Step \#2

Step \#3
Increased risk of Diabetes, Obesity<smiles>CCOC</smiles>

Worsens diabetes control

$\begin{aligned} & \text { glucose variability } \\ & \text { hypertension } \\ & \text { obesity }\end{aligned} \longrightarrow \begin{aligned} & \text { Inflammatory condition } \\ & \text { predisposing to } \\ & \text { atherosclerosis }\end{aligned}$

Induces an inflammatory state<smiles>CO</smiles>

Acute oxygen deprivation
Cardiovascular

catastrophe

Figure 1: The 3 steps hypothesis of the impact of OSAS on cardiovascular damage.

recording we also demonstrated that the presence of OSAS is associated with an abnormally increased instability of the fasting blood glucose [20]. The essence of these studies is that any disturbance of sleep architecture and/or presence of OSAS may interfere with multiple parameters of the blood glucose and of the blood pressure control.

We hypothesize that OSAS may have multiple loci of impact on the metabolic disorders. On one hand the respiratory disturbance may precipitate or worsen obesity, Diabetes and hypertension, with their ominous consequences. This has been widely demonstrated, and we have recently added another dimension to the list, that is the glucose variability, which is probably not less or even more dangerous than fixed hyperglycemia [21]. On the other hand OSAS, causing acute oxygen deprivation, increases the risk of acute cardiovascular death in the diabetic/obese subject with existing cardiovascular disease and arterial stenosis. In this paper we focus on this lethal outcome [22].

\section{OSAS as a driver for fatal cardiovascular events in the general population}

There are relatively few reports in the literature of an acute lethal effect of OSAS in the general population [23-25]. Many recent studies demonstrate an increased risk for all cause mortality [26-29]. All these studies however were addressed to a clinic referred population, which may represent a bias, and give limited information on the coexistent risk factors. Contrary to the others, the Busselton Study [22] included community based subjects. None of the 295 subjects who completed the study had diabetes at baseline, and 9 incident cases of diabetes within 4 years. Subjects with moderate to severe sleep apnea had a fully adjusted risk ratio for mortality of $6.24(\mathrm{p}=0.002)$. In another study after adjustment for age, sex, race, smoking status, alcohol-consumption, body-mass index, the presence or absence of diabetes mellitus, hyperlipidemia, atrial fibrillation, and hypertension the HR $(95 \% \mathrm{CI})$ for cardiovascular mortality was $5.2(1.4,19.2)$ in severe OSAS versus non OSA, [24]. In 2008 the results of a 18 year follow-up survey of a wide random sample $(n=1522)$ of the Wisconsin community were published [24] and demonstrated a significant increase in cardiovascular mortality with severe OSAS (Adjusted HR=5.2). Noteworthy among 1522 study participants only 50 had a diagnosis of diabetes, thus any conclusion on this specific population appears non-warranted. Another prospective study demonstrated Hazard Ratio for cardiovascular disease of 2.25 (CI 1.41 to 3.61) for those with severe OSAS in a cohort of 939 elderly subjects, an age with high prevalence of diabetes [30].

\section{OSAS as driver for fatal cardiovascular events in people with DM or other CVR factors}

Surprisingly very few of the studies on the effect of OSAS included critically ill subjects or those with extensive cardiovascular damage, a group where the acute reduction of oxygen delivery is likely to precipitate a serious ischemic event. Diabetes is a paradigm of this condition. Diabetics have extensive cardiovascular damage already evident at a young age. According to our hypothesis OSAS may have multiple loci of impact on the vascular catastrophe of diabetes: (1) as a cofactor predisposing to the disease $[31,32]$ (2) worsening the prognosis of the disease through multiple mechanisms, including the disregulation of blood glucose control, a persistent inflammatory condition [3335] and obesity [36-39]. This last condition is also dependent on reduced sense of satiety at least partially mediated by the hormone Leptin [40] which is regulated by sleep and is suppressed 
in OSAS subjects [40] (3) inducing serious oxygen deprivation as the final step on an already compromised cardiovascular system. However we could not find studies of OSA effect on the appearance of death, AMI, Stroke in a high risk population with pre-existent cardiovascular disease. The most common reason for intensive care unit admission in critically ill OSA patients seems to be a cardiovascular event, followed by respiratory and cerebrovascular events [41]. Although understanding of hypoxic pulmonary vasoconstriction (HPV) has advanced significantly, major areas of ignorance and uncertainty still await resolution: for the last 20 years, it has been clear that the essential sensor, transduction, and effector mechanisms responsible for HPV reside in the pulmonary arterial smooth muscle cell and have strong impact upon heart myocardial function and coronary perfusion [42]. In addition to that, aortic dilation secondary to OSA-driven blood pressure increase [43] further aggravates cardiovascular disease. In patients with severe OSA increased afterload from systemic hypertension rather than mechanical stress on the aortic wall determines thoracic aortic size and causes abnormalities in aortic function indexes [44].

\section{Mechanisms underlying increased cardiovascular mortality rate in OSAS patients}

The idea that oxygen deprivation may acutely precipitate a cardiovascular event, although suggestive, has not been clearly proven till now. Of course such demonstration is very difficult if not impossible to obtain, except than under experimental conditions that would be unethical for the human and the animal in general. Nevertheless some support to our hypothesis comes from a sophisticated observational study performed through an international cooperation in 7 OSAS patients by using transcranial doppler sonography (TCD) and polysomnography [45]. During this experiment TCD measurements of cerebral blood flow velocity (CBFV) showed a regular, undulating pattern with flow minima immediately before apnoeas or hypopnoeas and maxima closely after their termination, reciprocally to peripheral 02 saturation. CBFV reactivity was significantly diminished in consecutive respiratory events (CRE) compared to non-CRE periods. On the other hand, phase differences in cerebral pulse transit time - defined as the speed of the pulse wave travelling from the heart to the middle cerebral artery and reflecting vascular stiffness - were reduced in non-CRE, and even more so in CRE periods, compared to initial wake phases. These data suggest the existence of impaired vessel reactivity and increased arterial stiffness especially during periods characterized by many consecutive respiratory events also lends strong support to the possible occurrence of severe disturbances of cerebral circulation in OSA patients and more in general of endothelium dysfunctional changes. It has been recognized that cerebral hypoxia occurs in untreated OSA, causing an increase in the arousal threshold, which may predispose critically ill patients to prolonged apnoeic episodes and death during sleep [46].

Mortality rate is expected to be increased by the presence of the heavy OSA burden. This view is strongly supported by a recently published large polysomnography-based investigation showing that OSA predicted incident sudden cardiac death (SCD) independently of well-established risk factors [47]. A smaller follow-up study of 89 patients undergoing PCI for acute coronary syndrome came to similar conclusions as multivariate analysis pointed to OSA as independent predictor for major adverse cardiac events, with a hazard ratio as high as 11.6 [48].

Actually OSA may inhibit the recovery of left ventricular function in patients with AMI, [49] which in turn worsens OSA, thus triggering a deadly vicious circle [50]. The opposite mortality trend had been observed after CPAP treatment. In fact impairment of endothelial-dependent vasodilation correlates with the degree of endothelial cell apoptosis in patients with OSA. In these subjects CPAP therapy leads to a significant decline in circulating apoptotic endothelial cells [51]. This provides a convincing pathophysiological mechanism acting at the local level, which may explain at least partially the promising results obtained with CPAP on clinical grounds. An interesting review of prospective observational cohort studies, found that in patients with severe OSAS CPAP confers protective effects against death from cardiovascular disease by reducing the risk of fatal and nonfatal cardiovascular outcomes and overall mortality, and also identified indirect evidence for stroke reduction [52]. Unfortunately adherence to prescribed CPAP therapy is extremely low both at home and during the hospital stay including the perioperative period, so that further research is needed to identify and overcome barriers to CPAP acceptance and adherence at least in the perioperative setting. We will overlook this phenomenon as it exceeds the scope of the present review [53].

\section{OSA as a life-threatening factor in patients with $\mathrm{HF}$}

Sleep-disordered breathing (SDB), including OSA, is highly prevalent and frequently unrecognized in patients with chronic heart failure (HF) who are at high mortality risk per se. Untreated SDB may precipitate acute HF and delay recovery by increasing vascular inflammation and oxidative stress, impairing control of blood pressure, and promoting arrhythmias. Actually OSA has been proposed as cardiovascular disease itself and precipitating factor for HF. Untreated OSA doubles the risk for developing $\mathrm{HF}$, and patients with HF and OSA have a worse prognosis than patients with $\mathrm{HF}$ alone through increased afterload, intermittent hypoxia, and excess sympathetic activity which weaken the already failing heart [54]. Similar to the findings in the general population, treatment of OSA appears to reduce cardiovascular morbidity and mortality in HF, so that CPAP has been proposed as an efficacious therapy $[55,56]$. To the contrary, CPAP withdrawal is associated with prolongation of the indexes of cardiac repolarization and dispersion of repolarization, which may provide a possible mechanistic link between OSA, cardiac dysrhythmias, and sudden cardiac death [57].

\section{Hypoxic events as risk factor for arrhythmic emergencies}

Acute hypoxic respiratory failure due to non-cardiogenic pulmonary edema with different etiology displays high mortality (about 21\%) [58] In fact OSA results in multiple intermediate 
mechanisms that may promote atrial fibrillation, ventricular arrhythmias, and sudden cardiac death. These mechanisms may act acutely to trigger nocturnal dysrhythmias, or chronically by affecting the electrical and myocardial substrates. Numerous epidemiological data have identified increased risk for atrial fibrillation and sudden cardiac death related to OSA [59]. This is even more critical when dealing with patients undergoing surgical procedures. In patients with clinical features of OSA, the number of hypoxic episodes determined by home nocturnal oximetry, is associated with an increased rate of postoperative complications [60]. Because of this a series of surgical perioperative and internal medicine ward oriented protocols are developed every now and then in order to prevent in-hospital sudden deaths in critical patients [61].

\section{The three-step hypothesis for diabetic patients}

To our knowledge the three-step hypothesis has not been put forward with respect to people with diabetes. At present we must rely on epidemiological data, which present a gross margin of error. The diabetic fragile patient has many other ongoing and concurring problems, like atherosclerosis, anemia, drug treatments, and kidney failure. On the other hand diabetes per se does not seem to increase the mortality rates of cardiac surgery despite a 3- to 5-fold increase in renal, neurological, haematological and even pulmonary complications [62] Mortality within ICU's is increased by marginal 1.2 times, mostly in case of kidney complications [63], although hyperglycemic patients, irrespective of a previous diagnosis of diabetes, have a higher mortality than euglycemic ones [64]. After taking together all these considerations, we are convinced that in people with diabetes one true killer is OSA, potentially masked and made more dangerous by its typical comorbidities, including diabetes with its accompanying features, like high glucose variability or persistent hyperglycemia. Actually it is difficult to dissect the life-threatening consequences of OSA, cardiovascular disease and diabetes from one other, and this may be the reason why cardiovascular mortality has been mostly ascribed to glucose metabolism defects to date. This paper represents a hypothesis built on the current literature and not on a sound experimental/ observational basis. However any science is built on a theory, and this paper, with its limitations, aims to be the basis for a research model that can demonstrate over the long haul the real role of OSAS as a serious culprit for the high cardiovascular mortality rate in diabetes.

\section{References}

1. Stamler J, Vaccaro O, Neaton JD, Wentworth D (1993) Diabetes, other risk factors, and 12-yr cardiovascular mortality for men screened in the multiple risk factor intervention trial. Diabetes Care 16(2): 434444.

2. Boussageon R, Bejan-Angoulvant T, Saadatian-Elahi M, Lafont S, Bergeonneau C, et al. (2011) Effect of intensive glucose lowering treatment on all cause mortality, cardiovascular death, and microvascular events in type 2 diabetes: meta-analysis of randomised controlled trials. BMJ 343: d4169.

3. Yudkin JS, Richter B, Gale EA (2010) Intensified glucose lowering in type 2 diabetes: time for a reappraisal. Diabetologia 53: 2079-2085.
4. ACCORD Study Group, Gerstein HC, Miller ME, Genuth S, Ismail-Beigi $\mathrm{F}$, et al. (2011) Long-term effects of intensive glucose lowering on cardiovascular outcomes. N Engl J Med 364: 818-828.

5. Hirsh IB, Brownlee M (2005) Should minimal blood glucose variability become the gold standard of glycemic control? J Diabetes complications 19(3): 178-181.

6. Resnick HE, Redline S, Shahar E, Gilpin A, Newman A, et al. (2003) Diabetes and sleep disturbances: findings from the Sleep Heart Health Study. Diabetes Care 26: 702-709.

7. Einhorn D, Stewart DA, Erman MK, Gordon N, Philis-Tsimikas A, et al. (2007) Prevalence of sleep apnea in a population of adults with type 2 diabetes mellitus. Endocr Pract 13: 355-362.

8. Aronsohn RS, Whitmore H, Van Cauter E, Tasali E (2010) Impact of untreated obstructive sleep apnea on glucose control in type 2 diabetes. Am J Respir Crit Care Med 181: 507-513.

9. Foster GD, Sanders MH, Millman R, Zammit G, Borradaile KE et al. (2009) Obstructive sleep apnea among obese patients with type 2 diabetes. Diabetes Care 32: 1017-1019.

10. Muraki I, Tanigawa T, Yamagishi K, Sakurai S, Ohira T et al. (2010) Nocturnal intermittent hypoxia and the development of type 2 diabetes: The circulatory risks in communities study (CIRCS). Diabetologia 53: 481-488.

11. Punjabi NM, Beamer BA (2009) Alterations in glucose disposal in sleep-disordered breathing. Am J Respir Crit Care Med 179: 235-240.

12.http://www.idf.org/webdata/docs/APNOEA_final.pdf

13. Cappuccio FP, D’Elia L, Strazzullo P, Miller MA (2010) Quantity and quality of sleep and incidence of type 2 diabetes: a systematic review and meta-analysis. Diabetes Care 33: 414-420.

14. Ayas NT, White DP, Al-Delaimy WK, Manson JE, Stampfer MJ, et al. (2003) A prospective study of self reported sleep duration and incident diabetes in women. Diabetes Care 26: 380-384.

15. Vgontzas AN, Liao D, Pejovic S, Calhoun S, Karataraki M, et al. (2009) Insomnia with objective short seep duration is associated with type 2 diabetes: a population-based study. Diabetes Care 32: 1980-1985.

16. Mallon L, Broman JE, Hetta J (2005) High incidence of diabetes in men with sleep complaints or short sleep duration: a 12-year follow-up study of a middle-aged population. Diabetes Care 28: 2762-2767.

17. Tatti P, Passali D (2012) Sleep Disturbances and glucose variability. In: CM Lopez Garcia \& PA Perez Gonzalez (Eds.), Handbook on Metabolic Syndrome. Nova Science publishers.

18. Marin J, Carrizo S (2007) Mortality in obstructive sleep apnea. Sleep Med Clin 2: 593-601.

19. Phillips B (2005) Sleep-disordered breathing and cardiovascular disease. Sleep Med Rev 9: 131-140.

20. Tatti P, Strollo F, Passali D (2013) Sleep apnea, sleep disturbance, and fasting glucose variability: a pilot study. J Diabetes Sci Technol 7(3): 743-748.

21. Monnier L, Colette C (2008) Glycemic variability: should we and can we prevent it? Diabetes Care 31: S150-S154.

22. McNicholas WT, Bonsigore MR; Management Committee of EU COST ACTION B26 (2007) Sleep apnoea as an independent risk factor for cardiovascular disease: current evidence, basic mechanisms and research priorities. Eur Respir J 29: 156-178. 
23. Pack AI et al. (2008) Does untreated sleep apnea lead to death? Sleep 31(8): 1067-1068

24. Marshall NS, Wong KK, Liu PY, Cullen SR, Knuiman MW, et al. (2008) Sleep apnea as an independent risk factor for all-cause mortality: the Busselton Health Study. Sleep 31: 1079-1085.

25. Young T, Finn L, Peppard PE, Szklo-Coxe M, Austin D, et al. (2008) Sleep-disordered-breathing and mortality: eighteen-year follow-up of the Wisconsin sleep cohort. Sleep 31: 1071-1078.

26. Yaggi HK, Concato J, Kernan WN, Lichtman JH, Brass LM, et al. (2005) Obstructive sleep apnea as a risk factor for stroke and death. N Engl J Med. 353: 2034-2041.

27. Marin JM, Carrizo SJ, Vicente E, Agusti AG (2005) Long-term cardiovascular outcomes in men with obstructive sleep apnoeahypopnoea with or without treatment with continuous positive airway pressure: an observational study. Lancet 365: 1046-1053.

28. Lavie P, Lavie L, Herer P (2005) All-cause mortality in males with sleep apnoea syndrome: declining mortality rates with age. Eur Respir J 25: 514-520.

29. Peker Y, Carlson J, Hedner J (2006) Increased incidence of coronary artery disease in sleep apnoea: a long-term follow-up. Eur Respir J 28: 596-602.

30. Martínez-García MA, Campos-Rodríguez F, Catalán-Serra P, SolerCataluña JJ, Almeida-Gonzalez C, et al. (2012) Cardiovascular mortality in obstructive sleep apnea in the elderly: role of long-term continuous positive airway pressure treatment: a prospective observational study. Am J Respir Crit Care Med 186(9): 909-916.

31. BotrosN, ConcatoJ, MohseninV, SelimB, Doctor K, et al. (2009) Obstructive sleep apnea as a risk factor for type 2 diabetes. Am J Med 122(12): 1122-1127.

32. Punjabi NM, ShaharE, Redline S, Gottlieb DJ, GivelberR, et al. (2004) Sleep-disordered breathing, glucose intolerance, and insulin resistance: the Sleep Heart Health Study. Am J Epidemiol 160(6): 521530.

33. Selmi C, Montano N, Furlan R, Keen CL, Gershwin ME (2007) Inflammation and oxidative stress in obstructive sleep apnea syndrome. Exp Biol Med (Maywood) 232(11): 1409-1413.

34. Steiropoulos P, Papanas N, Nena E, Antoniadou M, Serasli E, et al. (2010) Inflammatory markers in middle-aged obese subjects: does obstructive sleep apnea syndrome play a role? Mediators Inflamm 2010: 675320 .

35. Baessler A, Nadeem R, Harvey M, Madbouly E, Younus A, et al. (2013) Treatment for sleep apnea by continuous positive airway pressure improves levels of inflammatory markers - a meta-analysis. Journal of Inflammation 10: 13.

36. Wilcox I, McNamara SG, Collins FL, Grunstein RR, Sullivan CE (1998) 'Syndrome Z': the interaction of sleep apnoea, vascular risk factors and heart disease. Thorax 53: S25-S28.

37. Schwartz AR, Patil SP, Laffan AM, Polotsky V, Schneider H, et al. (2008) Obesity and obstructive sleep apnea: pathogenic mechanism and therapeutic approaches. Pro Am Thorac Soc 5(2): 185-192.

38. Taheri S, Lin L, Austin D, Young T, Mignot E (2004) Short sleep duration is associated with reduced leptin, elevated ghrelin, and increased body mass index. PLoS Med 1(3): e62.

39. Chin K, Shimizu K, Nakamura T, Narai N, Masuzaki H, et al. (1999) Changes in intra-abdominal fat and serum leptin levels in patients with obstructive sleep apnea syndrome following nasal continuous positive airway pressure therapy. Circulation 100: 706-712.

40. Patel SR, Palmer LJ, Larkin EK, Jenny NS, White DP, et al. (2004) Relationship between obstructive sleep apnea and diurnal leptin rhythms. Sleep 27(2): 235-239.

41. Hang LW, Chen W, Liang SJ, Lin YC, Tu CY, et al. (2010) Clinical characteristics and outcomes of patients with obstructive sleep apnoea requiring intensive care. Anaesth Intensive Car 38: 506-512.

42. Sylvester JT, Shimoda LA, Aaronson PI, Ward JP (2012) Hypoxic pulmonary vasoconstriction. Physiol Rev 92: 367-520.

43. Stradling JR, Pepperell J, Davies R (2001) Sleep apnoea and hypertension: proof at last? Thorax 56(Suppl II): ii45-ii49.

44. Lee LC, Torres MC, Khoo SM, Chong EY, Lau C, et al. (2010) The relative impact of obstructive sleep apnea and hypertension on the structural and functional changes of the thoracic aorta. Sleep 33: 1173-1176.

45. Furtner M, Staudacher M, Frauscher B, Brandauer E, Esnaola y Rojas MM, et Al. (2009) Cerebral vasoreactivity decreases overnight in severe obstructive sleep apnea syndrome: a study of cerebral hemodynamics. Sleep Med 10: 875-881.

46. Dyken ME, Yamada T, Glenn CL, Berger HA (2004) Obstructive sleep apnea associated with cerebral hypoxemia and death. Neurology 62: 491-493.

47. Gami AS, Olson EJ, Shen WK, Wright RS, Ballman KV, et al. (2013) Obstructive sleep apnea and the risk of sudden cardiac death: a longitudinal study of 10,701 adults. J Am Coll Cardiol 62: 610-616.

48. Yumino D, Tsurumi Y, Takagi A, Suzuki K, Kasanuki H (2007) Impact of obstructive sleep apnea on clinical and angiographic outcomes following percutaneous coronary intervention in patients with acute coronary syndrome. Am J Cardiol 99: 26-30.

49. Nakashima H, Katayama T, Takagi C, Amenomori K, Ishizaki M, et al. (2006) Obstructive sleep apnoea inhibits the recovery of left ventricular function in patients with acute myocardial infarction. Eur Heart J 27: 2317-2322.

50.Tsukamoto K, Ohara A (2006) Temporal worsening of sleepdisordered breathing in the acute phase of myocardial infarction. Circ J 70: 1553-1556.

51.El Solh AA, Akinnusi ME, Baddoura FH, Mankowski CR (2007) Endothelial cell apoptosis in obstructive sleep apnea: a link to endothelial dysfunction. Am J Respir Crit Care Med 175: 1186-1191.

52. Yaggi H, Mohsenin V (2007) Sleep apnea and stroke: a risk factor or an association? Sleep Med Clin 2: 583-591.

53. Guralnick AS, Pant M, Minhaj M, Sweitzer BJ, Mokhlesi B (2012) CPAP adherence in patients with newly diagnosed obstructive sleep apnea prior to elective surgery. J Clin Sleep Med 8: 501-506.

54.Brisco MA, Goldberg LR (2010) Sleep apnea in congestive heart failure. Curr Heart Fail Rep 7: 175-184.

55. Jelic S, Le Jemtel TH (2009) Sleep-disordered breathing in acute decompensated heart failure. Curr Heart Fail Rep 6: 169-175.

56. Devulapally K, Pongonis R Jr, Khayat R (2009) OSA: the new cardiovascular disease: part II: overview of cardiovascular diseases associated with obstructive sleep apnea. Heart Fail Rev 14: 155-164.

57. Rossi VA, Stoewhas AC, Camen G, Steffel J, Bloch KE, et al. (2012) The effects of continuous positive airway pressure therapy withdrawal on cardiac repolarization: data from a randomized controlled trial. Eur Heart J 33: 2206-2212. 
58. Lakhey S, Shrestha R, Thapa S, Tuladhar S (2010) Scenario of acute respiratory distress syndrome in a tertiary care center. JNMA J Nepal Med Assoc 49: 129-132.

59.Gami AS, Somers VK (2008) Implications of obstructive sleep apnea for atrial fibrillation and sudden cardiac death. J Cardiovasc Electrophysiol 19: 997-1003.

60. Hwang D, Shakir N, Limann B, Sison C, Kalra S, et al. (2008) Association of sleep-disordered breathing with postoperative complications. Chest 133: 1128-1134.

61. Bolden N, Smith CE, Auckley D (2009) Avoiding adverse outcomes in patients with obstructive sleep apnea (OSA): development and implementation of a perioperative OSA protocol. J Clin Anesth 21: 286-293.

62. Morricone L, Ranucci M, Denti S, Cazzaniga A, Isgrò G, et al. (1999) Diabetes and complications after cardiac surgery: comparison with a non-diabetic population. Acta Diabetol 36: 77-78.

63. Christiansen CF, Johansen MB, Christensen S, O’Brien JM, Tønnesen E, et al. (2013) Type 2 diabetes and 1-year mortality in intensive care unit patients. Eur J Clin Invest 43: 238-247.

64. Leite SA, Locatelli SB, Niece SP, Oliveira ARF, Tockus D, et al. (2010) Impact of hyperglycemia on morbidity and mortality, length of hospitalization and rates of re-hospitalization in a general hospital setting in Brazil. Diabetol Metab Syndr 2: 49. 